University of Nebraska - Lincoln

DigitalCommons@University of Nebraska - Lincoln

20th \& 21st Century French and Francophone Modern Languages and Literatures, Department Studies International Colloquium

$4-2020$

Représentation de la médina chez Namouss dans Le Fond de la Jarre d'Abdellatif Laâbi

Khanssaa Canning

Follow this and additional works at: https://digitalcommons.unl.edu/ffsc2020

Part of the Comparative Literature Commons, French and Francophone Literature Commons, and the Other French and Francophone Language and Literature Commons

This Presentation is brought to you for free and open access by the Modern Languages and Literatures, Department of at DigitalCommons@University of Nebraska - Lincoln. It has been accepted for inclusion in 20th \& 21st Century French and Francophone Studies International Colloquium by an authorized administrator of DigitalCommons@University of Nebraska - Lincoln. 
20th \& 21st Century French and Francophone Studies International Colloquium, University of Nebraska-Lincoln, March 26-28, 2020.

https://digitalcommons.unl.edu/ffsc2020/

DOI: $10.32873 /$ unl.dc.ffsc.027

Copyright (C) 2020 Khanssaa Canning

\title{
Représentation de la médina chez Namouss dans Le Fond de la Jarre ${ }^{1}$ d'Abdellatif Laâbi.
}

\author{
Khanssaa Canning \\ Alma College
}

Le Fond de la jarre d'Abdellatif Laâbi projette la médina de Fès sous une mise en scène totalement différente des anciennes représentations de ce même lieu par l'auteur, qui, depuis son expérience de l'incarcération de 1972 à1980, a pris des allures d'un espace dénaturé et impur. Une angoisse existentielle, une crise du Moi avait désormais habité Laâbi, depuis les années quatrevingt ; plus spécifiquement, deux ans après sa libération de huit ans et demi d'emprisonnement (1982). Son œuvre Le chemin des ordalies ${ }^{2}$ en dévoile les instances et interroge les contours de l'identité et de l'appartenance par rapport aux discours politique, sociologique et même philosophique au Maroc, ce qui fait que la problématique identitaire ne prendrait place chez lui qu'au croisement de ces discours dans leur ensemble.

A la crise du Moi et à l'aliénation identitaire s'ajoute la thématique du cloisonnement qui n'a cessé de résonner dans ses écrits jusqu'à la publication du Fond de la jarre où l'auteur cède la place à un regard plus atténué et moins tendu sur sa relation à cet espace d'origine. Cette œuvre est sûrement le passage le moins douloureux de la vie de Laâbi. Un récit qui pourrait être perçu comme un véritable « hymne » à la médina de Fès et qui viendrait s’ajouter aux récits des

\footnotetext{
${ }^{1}$ Abdellatif Laâbi, Le fond de la Jarre, Collection : FOLIO, France, 2006

${ }^{2}$ Abdellatif Laâbi, Les Chemins des ordalies, La Différence, Coll. « Minos », Paris, 2003.
} 
quêtes antérieurs laâbiennes (on a l'impression que ses œuvres antécédentes préparaient le terrain pour ce roman qui semble être écrit avec plus d'exultation.

Ainsi, l'auteur-narrateur-personnage nous raconte son enfance alors qu'il avait « sept ans, peut-être huit » (FDJ, p. 21) et nous emporte dans ses périples pour nous faire découvrir les multiples facettes de la vielle ville de Fès et de la vie d'un enfant qui évolue et qui apprend à se découvrir dans son environnement socioculturel et familial. Dans un métalangage où se mêlent innocence et imagination, réalité et rêverie, expériences et leçons à apprendre..., l'œuvre a la saveur de l'inouïe et de l'inattendu qui nous sont présentées par le biais d'une description panoramique des faits ranimant la médina.

Dans ce qui suit j'avance que Le Fond de la Jarre est une œuvre de réconciliation où la quête dite identitaire est tout à fait différente de celle à laquelle, lecteurs, on s'est habitué dans la littérature maghrébine d'expression française. A ce propos, j'ai eu la chance de poser la question personnellement à Laâbi : Parlez-vous d'une quête identitaire plurielle et collective dans Le Fond de la Jarre ? Il m'a répondu que :

«On ne peut parler de quête identitaire dans le Fond de la Jarre, contrairement à d'autres de mes livres où cette question est abordée, mais pas dans le sens où vous l'entendez. Mais il est vrai que, dans ce récit, il y a une tentative de sauvegarder une mémoire, une expérience humaine menacées par l'oubli. Mon ambition était peut-être de démontrer que cette petite partie de l'humanité au de laquelle j'ai vu le jour, grandi et me suis accompli peut faire accéder celui qui la découvre (et partout dans le monde) à l'expérience humaine dans toute sa complexité, avec ses souffrances et ses joies, ses doutes, ses révoltes, ses 
larmes et ses rires, ses petitesses et se grandeurs. Et c'est ainsi qu'en tout temps la littérature parvient à inscrit le particulier dans l'universel $»^{3}$

Pour l'auteur, du particulier à l'universel s'inscrit donc une continuité. Ainsi Le FDJ est-elle une œuvre où tiennent une écriture et un style cherchant à faire ressusciter, par les fragments du passé que la mémoire a su longtemps préserver, un vécu, une expérience humaine, un patrimoine, une cuture et un imaginaire qui tous s'entrelacent et se lient dans l'espace natal et où les personnages et les faits s'aboutissent par une cérémonie des confidences tout en évitant soigneusement les chausse-trappes, les images et imageries, les clichés et lieux communs qui encombrent volontiers ce type de livres qui fleurent parfois l'exotisme folklorique, l'ennui et le stéréotype.

L'auteur parvient à insuffler une dimension populaire non folklorisée à son œuvre se démarquant par là des récits dits ethnographiques, mais aussi du piège de l'orientalisme. Laâbi articule ainsi son récit autour de Namouss, l'enfant-personnage, qui pourtant se tient comme un oxymoron identitaire pour l'écrivain : il lui est en même temps complice et distant, Namouss, comme il le dit, «c'est mon ancêtre et mon enfant » et c'est dans l'entente de cette relation ambigüe que l'auteur, narrateur-héros nous invite à explorer avec lui une vie de ses secrets, d'apprentissage, de joie et des fois de peine et de souffrances.

Dès le début l'auteur s'assure de nous avertir que «c'est la prime enfance qui sera revisitée » et nous informe que l'impasse sera faite sur les thèmes tant répétés chez les écrivains coloniaux et ceux qui ont suivi leur voie : Ainsi l'école coranique, la cérémonie de la circoncision, la fête du mouton, le Hammam, et l'autoritarisme paternel (la famille patriarchal) sont-ils déjoués pour une

\footnotetext{
${ }^{3}$ Notre entretient avec lui en 2007
} 
volenté de donner à la réalité socio-culturelle une vision de l'intérieur en opposition avec les représentations mythiques et idéologiques qui ont marquées l'expression identitaire d'une littérature ethnographique en évitant les procédés qui rappellent le roman exotique comme l'insistance sur le pittoresque dont la visée implique tout lecteur étranger à la culture marocaine.

Par le biais de l'autofiction ${ }^{4}$ l'auteur s'attache à évoquer la vie quotidienne d'une famille populaire dans la médina de Fès, (nous sommes frappés par la diversité des réalités qui y sont évoquées), où on relève une authenticité et une fraîcheur que lui permet la focalisation par le regard d'enfant. Nous sommes invités à faire la connaissance de l'enfant de la médina, vivre son aventure et dévoiler cette pensée véhiculée par les constances de la rêverie cédant parfois la parole au narrateur-adulte et à son esprit critique intransigeant.

Chercher toutefois à classifier l'œuvre lâabienne sera une tâche pénible. Laâbi s'y détache carrément et tend à rejeter l'idée dans son ensemble : quand je lui ai posé la question, sa réponse était :

"Dans ma conception de la littérature, je n’ai jamais accepté cette séparation étanche qui a été instaurée entre les genres littéraires. J'ai toujours auvré pour faire communiquer les genres entre eux. La littérature n'évolue pas si on n'invente pas de nouvelles formes $\iota^{5}$.

Le Fond de la Jarre se voit comme un livre de transgression dont l'entreprise n'est point de falsifier le réel pour un souci de déformation ou transformation, il est plutôt question ici de faire part d'une

\footnotetext{
${ }^{4}$ A expliquer oralement la nuance de ce genre chez l'écrivain

5 “entretien avec Laâbi”, 2007
} 
nouvelle vision, laquelle, qui pour la première fois, réconcilie l'écrivain avec son passé dans l'attention d'éviter le cliché et l'étiquetage exotique.

\section{$\underline{\text { La médina : espace d'apprentissage et expression de l'intime }}$}

Le Fond de la jarre est en effet une sorte d'hommage à la médina, une reconnaissance d'un lieu qui fait le bilan d'une enfance et raconte les premières journées de Namouss (qui veut dire moustique vu sa santé fragile) à l'école, sa passion pour la langue française, la maison de la Sources des Chevaux, celle du quartier Siaj, les gens de la médina, leurs habitudes, son penchant pour la contemplation et la rêverie...etc. Elle se présente comme un univers mystérieux, un espace labyrinthique aux découvertes infinies ; Mais elle est surtout et avant tout l'expression d'un moi et le résultat d'une représentation littéraire dont l'intensité symbolique embrasse une nouvelle dimension ; il nous le dit d'ailleurs quand on lui a posé la question sur la raison de de cette longue attente pour écrire Le Fond de la Jarre "Vous avez écrit un roman qui vous écrit, pourquoi il a fallu attendre tout ce temps-là, surtout que vous vous êtes centré sur la période de votre enfance? » Et sa réponse était : «Le Fond de la jarre est ce que l'on peut appeler une œuvre de maturité. Si j'ai tardé autant à l'écrire, c'est que je n'étais pas satisfait par l'abondante littérature autobiographique écrite avant moi. Mon souci était de renouveler le genre, et de renouveler aussi mon écriture. Comme chaque fois que j'écris un nouveau livre, c'était un autre défi que je voulais relever $\gg{ }^{6}$

Ainsi, le récit introduit la médina comme génératrice d'une expression de l'intime où la relation entre le moi et l'espace s'engendre par une volonté d'associer les deux instances où la médina est comme un lieu de négociation identitaires. Dans un ton amusant et tendre, l'auteur nous

\footnotetext{
6 "entretien avec Laâbi"
} 
expose les bribes d'un passé que la mémoire d'un enfant a pu sauvegarder avec une focalisation majeure sur l'histoire personnelle (qui se lient indirectement aux évènements de l'époque). Par un va et vient entre le point de vue de l'auteur-narrateur adulte et celui de l'enfant, nous sommes invités à découvrir l'univers du protagoniste : un univers solitaire qui nourrit la curiosité d'un garçon prenant comme parcours d'apprentissage les aventures, les errances et les découvertes qui se déroulent dans les ruelles labyrinthiques de l'ancienne ville. Namouss sait parfaitement que vouloir aller au fond des choses c'est risquer de s'exposer à des mélancolies, des ennuis, des platitudes et à des désagréments, mais c'est au même temps toucher à des vérités qui ne sont pas toutes bonnes à dire : c'est en effet ce qui intrigue la curiosité de l'enfant qui semble à la recherche de quelque chose qui lui manque. La médina comme lieu d'expériences, d'aventure, de questionnement et de mystères se présente justement comme un espace d'adaptation et d'appropriation où l'enfant peut se questionner aussi bien qu' interroger les choses autour de lui:

« Et il ne sait quoi au juste. L’école ? [...]. Le voyage? [...]. L'amour des parents ? [...], Les amis ? [...]. Alors quoi ? peut-être une âme sæur à qui il pourrait confier son lourd secret [...] ou moins les idées bizarres qui lui passent par la tête, les images incompréhensibles qui défilent devant ses yeux dans son rêve éveillé, les vois qu'il entend, venant, on dirait d'un autre monde... » (p.150)

Namouss «rêve le monde et le monde existe comme il le rêve ». Entre le penser et le sentir la rêverie tient place et se mêle avec le réel. Le monde immédiat est transcendé et l'enfant « $a$ l'impression que son corps est soulevé par une force inconnue » (p.75) qui le projette dans une autre dimension de la solitude et de l'imagination; deux grands facteurs qui vont engager le futur écrivain dans la voie de l'écriture. 
Par ailleurs, quand il n'est pas aspiré dans son univers de rêves et d'imagination, Namouss entreprend de nouveaux périples avec de nouvelles aventures. L'apprentissage chez lui se fait dans les différents coins de la médina, à la sortie de la maison familiale au quartier, en passant par l'école et en circulant dans les petites ruelles. L'auteur nous présente donc l'espace natal à travers des coins et des sites appropriés psychologiquement pendant l'enfance, lesquels se réduisent (comme nous l'avons signalé) aux frontières du quartier de la résidence familiale. Des lieux accessibles et apprivoisés :

«Le territoire de Namouss est un mouchoir de poche. Il se limite au quartier des Kairouine, et encore. Quant à l'autre quartier, celui des Andalous, Namouss n'y a pour ainsi dire jamais mis les pieds. Pour lui, comme pour la plupart de ses camarades, c'est presque un pays étranger où il ne fait pas bon de s'aventurer. Là-bas vivent des bandes d'enfants hostiles qu'on ne rencontre que lors de batailles sporadiques » (p. 94)

\section{L'antithèse de la médina}

La médina dans Le Fond de la Jarre s'avère comme un paradoxe spatial, elle est un lieu de libres courts et de l'ouverture mais aussi un espace bordé et clos qui rappelle la demeure familiale. Pour désigner cet espace, l'auteur recourt au vocabulaire usuel ; aux toponymes s'ajoutent des termes du lexique commun du dialecte marocain, la langue arabe ou le français.

Au panorama des personnages s'ajoutent les sites et des endroits que Namouss visite durant ses pérégrinations et ses errances ; on y découvre alors les lieux sacrés (le sanctuaire de Moulay Idriss), les quartiers de commerce (Nejarrine), les espaces de divertissement (Les jardins de Jnane Sbil) et d'autres sites d'arrêt et du passage quotidien. Ces coins-là prennent de nouvelles significations chez l'enfant, ils deviennent une continuation, un écoulement créatif de nouveaux 
sens. La métaphore de déplacement est donc utilisée ici comme un procédé de création qui par extension s'applique à l'espace révélant un travail minutieux qui se répand dans tout le roman et qui se trouve enfin marqué par l'omniprésence de la médina.

Que Namouss puisse se représenter dans ce lieu ou s'y retrouver, voilà qui assure sans doute l'un des fondements de sa représentation personnelle de l'espace et situe d'emblée son fonctionnement psychique dans l'imaginaire spatial. C'est que, les images qui nous parcourent se configurent avant même que l'on tente de les inscrire dans la référence symbolique du langage. Peut-être qu'ici l'imaginaire de l'écrivain s'allie à un passé (la médina de l'enfance) qui revient l'habiter sous une autre forme, celle du souvenir. C'est pour ainsi dire que les constructions symboliques du passé (plutôt que le passé lui-même) sont vigoureusement structurées et profondément contraignantes : elles se gravent dans notre sensibilité à la manière des informations génériques. Le cas de la médina, en tant qu'espace du vécu, se représente enfin chez Namouss comme un lieu d'appropriation. Son exploration se varie entre le général et le particulier, l'ensemble et le détail, entre l'extérieur et l'intérieur tout en dépendant de l'état d'âme de l'enfant.

Paradoxalement, la ville natale se présente dans le roman comme un lieu d'errance qui se caractérise par l'ici et l'ailleurs. Le vagabondage de l'enfant y exprimé par des vocables tels : «l'aventure », « force inconnue », « autre dimension », « autres horizons », « être loin » .. aussi bien que les vocables spatiaux qui marquent les différents déplacements, physiques et mentales, de Namouss. De plus, les expressions qui ponctuent le roman représentent un véritable itinéraire géographique de l'errance formant ainsi le principal discours chez le narrateur-enfant; des expressions telles «liberté », « déplacement», « circuler », " pérégrinations », « errer », « découvrir », ...etc, témoignent les différents périples de Namouss qui sillonne la médina de bout 
en bout. Le désire de l'enfant d'explorer le lieu d'origine exprime cette quête incessante d'un espace perdu - peut-être- à jamais. Un sentiment nostalgique du narrateur adulte nous le dit :

«Le désir [...] s'impose à lui. Découvrir [Fès] avec les yeux qui ont voyagé, avec le besoin de garder en mémoire ce qu'il risque de perdre si jamais des ailes lui poussaient réellement et l'emmenaient si haut et si loin qu'il atteindrait un point de non-retour [...], il attendait avec conscience le moment où il allait percer la douce enveloppe et se tenir au seuil de la lumière. Et c'est la traversé qui commence. " (p.93)

De ce fait, la dimension micro-macrosome de la médina accentue à la fois une intimité, dont les grandes murailles appuient plus encore cette intelligence du secret et de l'intimité, mais aussi une extériorité, qui se donne à voir par son immense ciel ouvert aussi bien que les grands portails qui ne cessent de séduire Namouss par les secrets et les mystères au-delà des frontières murées. Cet espace clos et ouvert, immense et limité qui libère et enferme, fait-il de Namouss un être aliéné dans la logique du temps et de l'espace ? Un lieu de l'inouï et de l'inédit qui abrite des personnages différents «hors du commun qui le fascinent. Qui sont-ils? Anges ou diables? Mendiants ou prophètes? Allez savoir.» (p.95).

Labyrinthe, rêverie, dimensions inconnues, secrets et mystères ...Cette terminologie ajoute une tout autre dimension, imaginaire, presque mythique. Laâbi, qui n'hésite pas à confirmer ce mariage entre les deux extrêmes (réalité et fiction), et n'hésite non plus à lier l'imaginaire le plus exalté avec le réalisme le plus simplifié, explique :

«Le Fond de la Jarre est une œuvre littéraire. Elle recourt donc pour une part au réel, et pour l'autre à l'imaginaire [...] Dans mon livre, la réalité est « travaillée », 
transfigurée. Elle passe à travers le filtre des personnages, leur regard propre, leur psychologie, les liens qu'ils entretiennent entre eux, le rapport qu'ils ont à la période historique dans laquelle ils viennent $»^{7}$

Conclusion :

La médina de Fès, cet espace labyrinthique où l'identité d'une société s'engendre avec elle celle d'un enfant qui découvre la vie par un parcours d'apprentissage à travers les rencontres et les expériences. C'est l'espace des marchands, des artisans, des « maâllemine » et des « mtâalmine », celui des rhéteurs et de la parole sage, c'est là où la dignité collective avait comme seul prix le sang des marocains dont témoigne chaque ruelle et minaret. Le Fond de la Jarre vient ainsi rassembler ces instances dans un tableau qui réunit le vécu et l'histoire par une autofiction traçant une enfance particulière et universelle. Le lieu natal est revisité encore une fois mais avec beaucoup moins d'intensité et de tension.

Le sentiment de colère se calme finalement et l'attitude fort révolutionnaire s'apaise. Le ton est moins aggravé jusqu'au point de la plaisance. Et la médina, qui auparavant « dénaturée ${ }^{8}$ est désormais célébrée. Ce ton scelle "la fin d'une littérature de l'intériorité vécu comme un traumatisme $»^{9}$. Le Fond de la jarre est si l'on peut dire, est un livre d'aboutissement, dans le sens où il est le résultant d'un long parcours qui souligne « ce parvenir à mettre bout à bout les deux parties tronçonnées de [son] être $»^{10}$. L'auteur a choisi de remémorer les événements de son passé et d'y ajouter des révélations intimes, les confidences d'une histoire personnelle dans un contexte

\footnotetext{
77 Le présent entretien avec A. Laâbi été élaboré par nous, le 3 octobre 2007autour de son œuvre Le Fond de La Jarre.

8 Ibid, p.5

${ }^{9}$ Ibid.

${ }^{10}$ A. Laâbi, Les Chemins des ordalies, La Différence, Coll. « Minos », Paris, 2003. p. 194
} 
où la culture nourrit encore un imaginaire collectif. Justement, au-delà du simple réalisme autobiographique, la représentation de la ville dans ce récit consiste en une exploration personnelle qui livre une perception de soi dans les mouvements de la vie en société dans un temps précis. De ce fait, les critères qui fondent la sélection des lieux comme la maison familiale, la rue, le souk Sekkatine, ...etc., ont une grande importance parce qu'ils sont liés à des expériences intimes qui ont marqué le narrateur, mais ils sont aussi des lieux où gît une culture à sauvegarder de l'oubli.

Khanssaa El Alami Canning PhD Alma College canningk@alma.edu

\section{$\underline{\text { Annexe }}$}

\section{Entretien avec Abdellatif LAABI}

Dans le cadre du séminaire "Culture, imaginaire et style", assuré Dr. Khalid HADJI, et dans lequel s'inscrit notre projet de fin d'étude, le présent entretien a été élaboré le 3 octobre 2007 avec l'écrivain Abdellatif LAABI autour de son œuvre Le Fond de la jarre.

\section{1 - Certains critiques qualifient Le Fond de la jarre d'être une œuvre documentaire que répondrez-vous à cela?}

- Non. "Le Fond de la jarre" est une œuvre littéraire. Elle recourt donc pour une part au réel, et pour l'autre à l'imaginaire. Le documentaire ne peut pas se permettre un tel dosage. Il se contente d'enregistrer ce qu'il observe dans la réalité. Dans mon livre, par contre, la réalité est "travaillée", transfigurée. Elle passe à travers le filtre des personnages, leur regarde propre, leur psychologie, les liens qu'ils entretiennent entre eux, le rapport qu'ils ont à la période historique dans laquelle ils vivent.

2 - L'autobiographie est un cas où auteur, narrateur et personnages se rejoignent ; or, Le Fond de la jarre en étant qu'autobiographie (puisqu'il remplit les critères de ce genre) 
remplace le "je" par le "il" et présente des extraits qui, à notre humble avis nous semblent invraisemblables ; pouvons-nous vraiment parler ici d'une vraie autobiographie?

- S'il y a beaucoup d'éléments autobiographiques dans mon livre, on peut parler aussi a son propos de "fiction autobiographique".

3- Pourriez-vous nous dire qu'elle est votre propre représentation de la mère ? (Parce que et ce, en général, l’image de la mère soumise dont la personnalité est tout à fait gommée sous l'autorité du père est l'un des clichés de la littérature maghrébine d'expression française, ici c'est le contraire).

- Quant à ma représentation du père et de la mère, il me faut dire que je ne l'ai pas inventée. Elle recoupe ce que j'ai vécu réellement. Et c'est vrai qu'elle tranche avec l'image dominante dans beaucoup de récits de ce genre écrits par des Maghrébins, où le père est un despote et la femme un être soumis. Mes parents étaient différents. C'est ma mère, par exemple, qui était "l'homme fort" à la maison. Quoi qu'il en soit, et malgré les difficultés vécues, comme dans toute enfance d'ailleurs, je dois dire que c'est la tendresse qui domine chez moi dans mon rapport à mes parents car, avec la conscience acquise, ce que je retiens d'eux, c'est le trésor de valeurs humaines qu'ils m’ont légué.

4- A la question : entre roman et autobiographie que choisir ? Que répondrez-vous ?

- "Ma réponse à cette question est formulée en partie dans la précédente. J'ajoute simplement que, dans ma conception de la littérature, je n'ai pas jamais accepté cette séparation étanche qui a été instaurée entre les genres littéraires. J'ai toujours œuvré pour faire communiquer les genres entre eux. La littérature n'évolue pas si on n'invente pas de nouvelles formes."

5 - Vous avez écrit un roman qui vous écrit, pourquoi il a fallu attendre tout ce temps-là surtout que vous vous êtes centré sur la période de votre enfance?

- “Le Fond de la jarre est ce que l'on peut appeler une œuvre de maturité. Si j’ai tardé autant à l'écrire, c'est que je n'étais pas satisfait par l'abondante littérature 
autobiographique écrite avant moi. Mon souci était de renouveler le genre, et de renouveler aussi mon écriture. Comme chaque fois que j'écris un nouveau livre, c'était un autre défi que je voulais relever."

\section{6 - Y a-t-il des personnages irréels ou plus exactement fictifs à $100 \%$ dans Le Fond de la jarre?}

- “Tous les personnages du livre ont existé. Mais ce sont les situations où ils se trouvent dans telle ou telle partie du livre qui ont été transformés."

7 - Avez-vous consciemment choisi de vous adresser à un public spécifique par l’insertion de quelques expressions arabes sans traduction dans le texte de votre roman?

- Non, je ne m'adresse pas à un public particulier, de façon exclusive. C'est vrai que certains clins d'œil dans mon livre seront davantage appréciés par les Marocains et échapperont peut-être aux non-Marocains mais, dans l'ensemble, et si j'en juge d'après les réactions des uns et des autres, ce dosage du français, et de l'arabe marocain a été perçu comme l'un des éléments qui ont donné toute sa saveur au récit.

8 - Une fois que vous avez terminé votre œuvre, vous est-il arrivé de changer certaines pages pour ne pas choquer le public, orientant par la suite le contrat de lecture qui vous lie avec le public ? Si c'est le cas, pouvons-nous dire que le lecteur peut influencer l'auteur?

- 'L'écriture d'un livre est une aventure dont on ne sait pas d'avance où elle va aboutir. Elle nous fait emprunter plusieurs chemins, et il arrive que certains d'entre eux s'avèrent sans issue. Il faut parfois faire marche arrière et se mettre en quête d'un nouveau chemin. C'est donc la dynamique propre à l'œuvre qui est déterminante et non la présence d'un lecteur suppose. Mon rapport à celui-ci est d'ailleurs dicté par ce que j'estime être ma liberté. Pas question pour moi de pratiquer l'autocensure. Ce serait la pire des choses pour un écrivain. Il ne doit rien taire de ce qui est humain. 
La littérature n'est pas faite pour accepter les choses comme elles sont, mais pour en bouleverser l'ordre. Elle dérange les idées toutes faites, la perception qu'on peut avoir de soi et du monde. Elle s'adresse par conséquent au lecteur disposé à partager avec l'auteur l'aventure de l'œuvre de création."

\section{9 - Parlez-vous d'une quête identitaire plurielle et collective dans Le Fond de la Jarre ?}

- "On ne peut parler de quête identitaire dans "Le Fond de la jarre", contrairement à d'autres de mes livres où cette question est abordée, mais pas dans le sens où vous l'entendez. Mais il est vrai que, dans ce récit, il y a une tentative de sauvegarder une mémoire, une expérience humaine menacées par l'oubli. Mon ambition était peut-être de démontrer que cette petite partie de l'humanité au sein de laquelle j'ai vu le jour, grandi et me suis accompli peut faire accéder celui qui la découvre (et partout dans le monde) a l'expérience humaine dans toute sa complexité, avec ses souffrances et ses joies, ses doutes, ses révoltes, ses larmes et ses rires, ses petitesses et ses grandeurs. Et c'est ainsi qu'en tout temps la littérature parvient à inscrire le particulier dans l'universel."'

\section{Bibliographie}

\section{Euvres d'étude :}

- Abdellatif Laâbi, Le Fond de la jarre, Gallimard, 2002 ; Folio, n 5104, 2010.

- Cette communication est basée en majoritaire sur mon entretien avec Abdellatif LAABI, élaboré le 3 octobre 2007 à Fès dans le cadre du séminaire "Culture, imaginaire et style", et dans lequel s'inscrit mon projet de fin d'étude du master Didactique de français et Interculturalité.

\section{Euvres de références :}

- Abdellatif Laâbi, Les Chemins des ordalies, La Différence, Coll. « Minos », Paris, 2003.

- Le fond de la Jarre, Collection : FOLIO, France, 2006.

- Les Rides du lion, Messidor, Paris, 1989. 
- Réflexions littéraires sur l'espace public marocain dans l'œuvre d'Abdellatif Laâbi, par Safoi Babana-Hampton. Summa Publications, INC, Birmingham, Alabama (États-Unis), 2008.

- Abdellatif Laâbi : un intellectuel tout simplement, ouvrage collectif dirigé par Bernoussi Saltani, revue Interculturel Francophonies, Alliance française, Lecce (Italie), 2010. 